\title{
Large-area suspended graphene as a laser target to produce an energetic ion beam
}

\author{
Nur Khasanah ${ }^{1}$, Nima Bolouki ${ }^{1}$, Tzu-Yao Huang ${ }^{1}$, Yi-Zhe Hong ${ }^{1}$, Wen-Liang Chung ${ }^{1}$, Wei-Yen Woon ${ }^{1}$, \\ Ching-Yuan $\mathrm{Su}^{2}$, and Yasuhiro Kuramitsu ${ }^{1}$ \\ ${ }^{1}$ Department of Physics, National Central University, No. 300, Jhongda Rd., Jhongli, Taoyuan, 320 Taiwan \\ ${ }^{2}$ Graduate Institute of Energy Engineering, National Central University, No. 300, Jhongda Rd., Jhongli, Taoyuan, 320 Taiwan \\ (Received 1 November 2016; revised 18 February 2017; accepted 8 May 2017)
}

\begin{abstract}
Proton radiography is a key diagnostics to measure and image the electric/magnetic field in laser-produced plasmas. A thin solid target is irradiated with an intense laser pulse to produce a proton beam. The accelerated proton can achieve higher energy with thinner target. In order to produce an extremely thin target, we have developed a large-area suspended graphene as a laser target for energetic ion sources. We describe the manufacturing process of the suspended graphene, and show the results of quality evaluations.
\end{abstract}

Keywords: plasma; laser; ion acceleration; radiation pressure acceleration; graphene

\section{Introduction}

We have worked on the so-called laboratory astrophysics, where space and astrophysical phenomena are experimentally simulated in laboratories with high-power lasers, such as plasma jet ${ }^{[1-4]}$, collisionless shocks ${ }^{[5-10]}$ and hydrodynamic instabilities ${ }^{[11,12]}$. We recently extended our research field to include the relativistic regime of laboratory astrophysics with intense laser pulses ${ }^{[13-15]}$. A magnetic field is an essential quantity in many experiments mentioned above on laboratory astrophysics, and also in space/astrophysical phenomena. Therefore, proton/ion radiography is the key diagnostics for the magnetic field measurements.

We have observed a turbulent electric field excited by Kelvin-Helmholtz instability associated with collisionless shocks in laser-produced plasmas ${ }^{[7]}$, and have also observed time evolution of electric/magnetic structure formation in counterstreaming laser-produced plasmas ${ }^{[9]}$ with proton radiography. In these experiments a thin ( $\mu \mathrm{m}$ order) aluminum or gold target was irradiated with a short pulse laser to produce energetic protons. High energy radiations are emitted in wide direction due to the interaction between the forced oscillating relativistic electrons by the laser electric field and the nuclei of target material. At relatively small laser facilities, such as the NCU $100 \mathrm{TW}$ laser facility ${ }^{[16]}$, the radiation protection can be a serious practical problem due to

Correspondence to: Y. Kuramitsu, Department of Physics, National Central University, No. 300, Jhongda Rd., Jhongli, Taoyuan, 320 Taiwan. Email: yasu@ncu.edu.tw the limited space and the floor strength of the building. For instance, at the NCU $100 \mathrm{TW}$ laser facility the total weight of laser system, compression chambers and experimental stations including existing radiation shieldings are already close to the limit of the floor. Although we would like to develop the proton/ion radiography for the electric/magnetic field diagnostics, there is little room for the new radiation shielding for the proton/ion radiography.

Focusing an ultra intense laser onto a solid target, high energy radiations such as X-rays and gamma-rays will be emitted due to the interaction between the forced oscillating relativistic electrons by the laser electric field and the nuclei of target material. The radiation is simply proportional to the number of encounters of the relativistic electrons to the atomic nuclei in the target. In order to suppress this, we need to reduce the number of atoms in the ionized volume. To this end, we reduce the target thickness and choose the low atomic number material. The suspended graphene is an ideal solution for this. Although making a free-standing nanometer thin target is technically challenging, a large-area free-standing graphene has been successfully produced ${ }^{[17]}$. Synthesis of graphene through chemical vapor deposition (CVD) is a favorable method to prepare large scale materials as a nanometer-scale sheet of carbon atoms ${ }^{[18-20]}$. On the other hand, further improvement on productivity and thermal cost of the CVD process are necessary to reduce the duration of heating and cooling processes. In order to make shorter processing time and to achieve higher energy efficiency, the rapid thermal CVD (RTCVD) has 
been employed. The merits of graphene are as follows: we can control the thickness easily, it can be flat and homogeneous over a large area $(\geqslant 100 \mu \mathrm{m})$ compared with the laser focal spots $(\sim 10 \mu \mathrm{m})$ and manufacturing costs are not expensive. The free-standing graphene are also potentially useful for many other applications. For instance, proton/ion acceleration with a very thin target is relevant to radiation pressure acceleration (RPA), which is considered to be more efficient than the conventional proton acceleration with thicker targets ${ }^{[21-27]}$.

Stable mass production of low atomic number freestanding ultra-thin targets with nanometer accuracy allows us to investigate ion acceleration such as the RPA in the future. We note that the production cost of suspended graphene is not expensive. We use the energetic ion beams to measure electric/magnetic field on laboratory astrophysics experiments.

\section{Method}

The graphene sample is prepared based on a CVD method, where the large-area and high crystalline graphene film can be synthesized. RTCVD is one of the possible methods based on rapid thermal process ${ }^{[28]}$. First, a copper foil is electro-polished, and then, it is cleaned with acetone and deionized water to remove the organic contamination. Due to the radiation natures of the heating mechanism, the RTA provides a fast ramping and cooling. The major thermal process for graphene growth follows three stages as shown in Figure 1(a): a fast temperature ramping to target that takes around $300 \mathrm{~s}$. At this time, temperature reaches to $980^{\circ} \mathrm{C}$. At the second stage, the temperature remains constant for graphene growth at $980^{\circ} \mathrm{C}$ for $2200 \mathrm{~s}$. Finally, a fast cooling to room temperature occurs within $400 \mathrm{~s}$. After the ramping stage, a graphene film is grown on the copper substrate with RTCVD. The gas flows of hydrogen and methane (as the carbon source) are 100 and $50 \mathrm{sccm}$, respectively, under the stable temperature and pressure of 1.5 torr. After the graphene growth, heating lamps of RTA are switched off to perform a fast cooling, while the gas flows of hydrogen and methane continue until cooling down to the room temperature.

After RTCVD growth, the graphene on copper is spincoated with polymethyl methacrylate (PMMA) to protect the graphene. Then the PMMA-coated graphene on copper is dipped into an $\mathrm{NaOH}$ aqueous solution with a constant current supply. Using this bubble method, we remove the copper from the PMMA-coated graphene. The delamination of graphene from copper is carried out by the hydrogen bubbles produced via electrolysis of water at the PMMA-coated graphene on copper cathode as shown in Figure $1(\mathrm{~b})^{[29,30]}$. Multi-layered graphene can be fabricated by repeating the above processes, and the number of layers

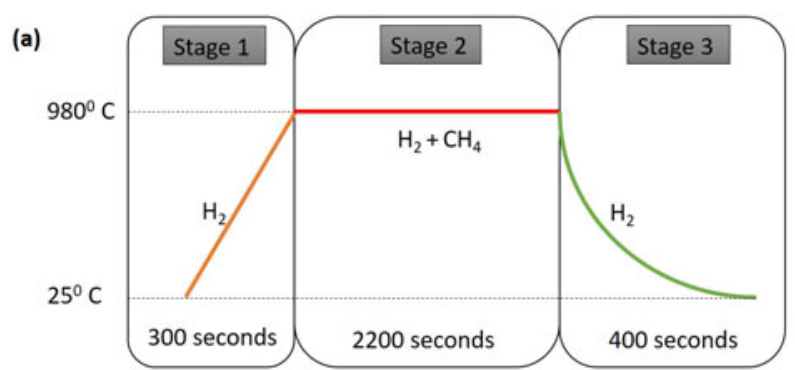

(b)

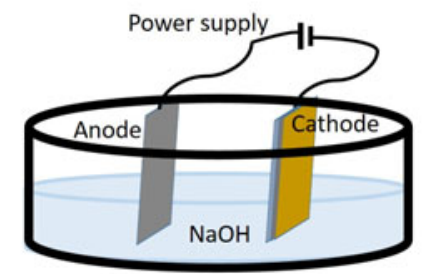

(c)

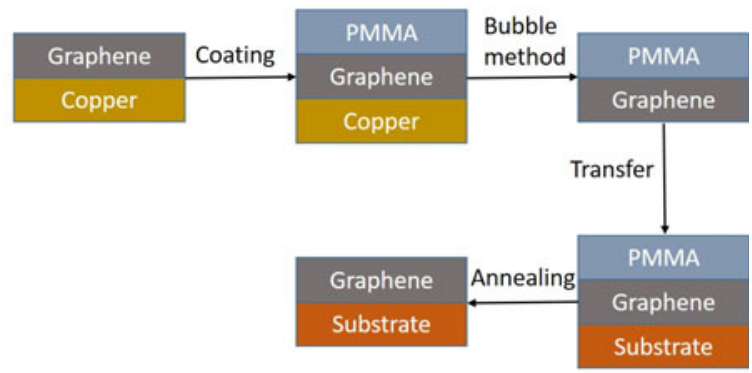

(d)

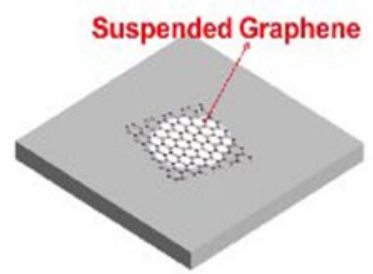

Figure 1. (a) Three-stage heating process of RTCVD, (b) Illustration of the bubbling transfer process of graphene from copper, (c) fabrication of suspended graphene, and (d) the illustration of suspended graphene on a substrate with a hole.

determines the thickness of the target, with a resolution of single carbon atom diameter. Then, single-layered or multi-layered PMMA-coated graphene is stamped onto the target substrate after cleaning with pure water ${ }^{[17]}$. After the stamping transfer process, we remove PMMA with $500^{\circ} \mathrm{C}$ annealing. We use a silicon chip as the substrate with a thickness of $250 \mu \mathrm{m}$, where there are many holes with diameter 100-500 $\mu \mathrm{m}$. Figure 1(d) shows a schematic image of a suspended graphene on a hole. Figure 1(c) shows the fabrication of suspended graphene. Raman spectroscopy has been used to ensure the existence of the suspended graphene film and characterize its properties. Typical Raman fingerprints of graphene include $\mathrm{G}\left(1580 \mathrm{~cm}^{-1}\right)$ and $2 \mathrm{D}$ $\left(2680 \mathrm{~cm}^{-1}\right)$ bands since Raman selection rules are always satisfied. Defects result in the appearance of the additional 


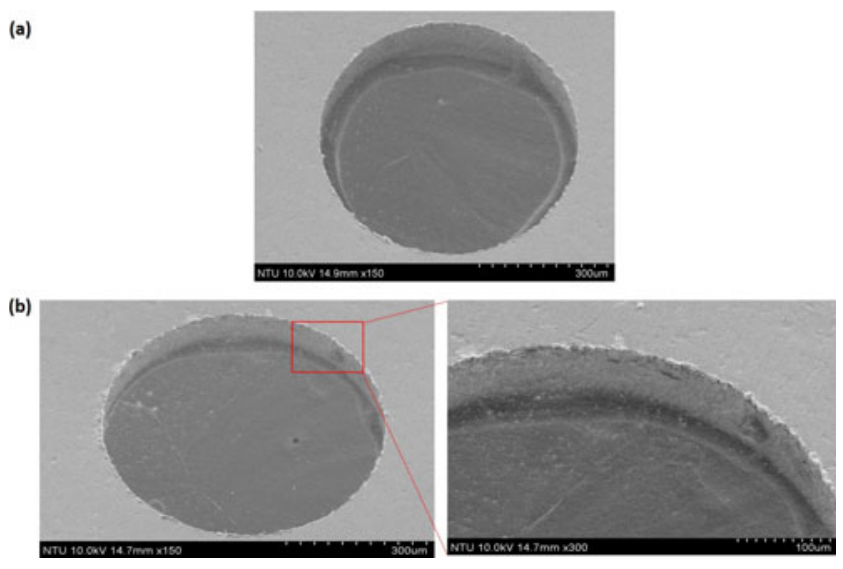

Figure 2. (a) The SEM images of SLG and (b) the SEM images of 4-L graphene film suspended on $500 \mu \mathrm{m}$ hole.

Raman forbidden D $\left(1350 \mathrm{~cm}^{-1}\right)$ and D' $\left(1620 \mathrm{~cm}^{-1}\right)$ bands as the defect provides the missing momentum to satisfy momentum conservation in the Raman scattering process. Namely, the D (D) band represents processes activated by a defect-assisted single-phonon intervalley (intravalley) scattering processes. A $532 \mathrm{~nm}$ excitation laser $(2.41 \mathrm{eV})$ is implemented in an optical microscope so that the spatial resolution of the measurement is about $1 \mu \mathrm{m}$.

\section{Results}

To detect impurities, ruptures, folds, voids and discontinuities of synthesized or transferred graphene on the substrate, we performed the scanning electron microscope (SEM) image on the suspended graphene. The SEM image of $500 \mu \mathrm{m}$ single-layered suspended graphene (SLG) is shown in Figure 2(a). This image indicates uniform film without contamination. Figure 2(b) shows an SEM image of $500 \mu \mathrm{m}$ 4-layered (4-L) suspended graphene. The edge of a ruptured sheet was selected to show both graphene and the silicon surface. A magnified view at the edge shows continuous wrinkles across the suspended and supported area, indicating the suspended graphene film is relatively flat across the hole boundary. The atomic force microscopy (AFM) in the non-contact mode has been employed to characterize the topography of the graphene samples. Figures 3(a) and 3(b) demonstrate the AFM height profiles. The horizontal and vertical axes show the distance $(\mu \mathrm{m})$ and height $(\mathrm{nm})$, respectively. The AFM images at the edge of an SLG is shown to demonstrate the height difference between the graphene surface and the substrate. The white line marks the cross-section across the graphene and silicon substrate with a height difference of around $1 \mathrm{~nm}$, suggesting an SLG film as shown in Figure 3(a), while the cross-section across the 4- $\mathrm{L}$ graphene is about $4 \mathrm{~nm}$ as shown in Figure 3(b). To determine the quality of the SLG and 4-L suspended graphene
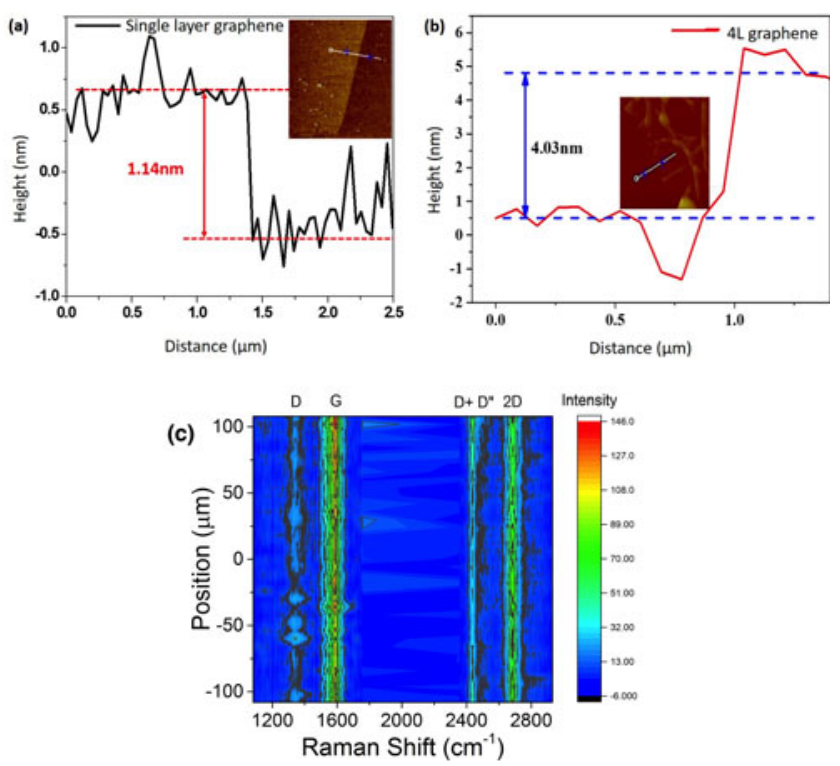

Figure 3. The AFM image and cross-section profile at the edge of (a) SLG and (b) 4-L suspended graphene film, respectively. (c) Raman spectra acquired along a line in a 4-L graphene suspended across a $250 \mu \mathrm{m}$ hole.

obtained, we performed Raman measurement on the suspended graphene [Figure 3(c)]. Both SLG and 4-L suspended graphene films exhibit low defect density (low D band), implying high crystallinity of graphene film. Figure 3(c) shows an example of Raman spectra acquired along a line on a four-layered graphene suspended across a $250 \mu \mathrm{m}$ hole. The $\mathrm{D}$ band is relatively low compared to the $\mathrm{G}$ band, indicating low defect density. The $2 \mathrm{D} / \mathrm{G}$ ratio is lower than 1 , indicating multiple layered graphene. Overall the similar intensities in the $G$ and $2 D$ show good uniformity of the suspended graphene in a large area.

The above data shows large area, controllable and relatively clean suspended graphene samples can be routinely prepared. This atomic thin graphene film with high purity and quality is expected to produce a controllable proton beam.

\section{Discussions and summary}

We have produced a large-area $(500 \mu \mathrm{m})$ suspended graphene as a target of energetic ion beam produced with an intense laser pulse, such as the NCU 100 TW laser facility. Based on SEM, AFM and Raman spectroscopy characterization, the surface flatness, thickness, i.e., the number of layers, and the quality of graphene have been confirmed.

We have produced suspended graphene on the substrate with many holes with different diameters. By using a 2axis motorized stage in the vacuum chamber and the target monitors, we can shoot many graphene targets without opening the chamber. This is still a slow process since we have to make sure of the target position at each shot. 
Although the repetition rate of the $100 \mathrm{TW}$ laser is $10 \mathrm{~Hz}$, the practical shot rate will be 1 shot per tens of seconds. In order to improve the shot rate, we need to use accurate and fast motional stage in future. Nevertheless, this is still better than the one-shot experiment, where we have to open the chamber to change the target after each shot; our graphene targets will save the limited laser machine time and increase the shot numbers, which results in improving the data and publication qualities.

The larger area of suspended graphene needs more careful treatment and is more difficult to manufacture. We test the optimum size for the ion target by using graphene with different diameters. The foot of a Gaussian short pulse is still intense; if the diameter is too small, the substrate will be ionized and may contaminate the experiments with the radiations.

Since the number of graphene layers and thus the thickness of the target is well controlled, the ion acceleration and its dependence on the target thickness can be investigated with high accuracy. For instance, when we use thin (but still $\mu \mathrm{m}$ order) targets to produce rear-side plasmas by irradiating with high-power lasers, the target thickness is one of the largest uncertainties. The graphene target can remove this uncertainty. Using the large-area suspended graphene targets, we investigate the RPA and use the energetic ion beams to measure electric/magnetic field on laboratory astrophysics experiments.

\section{Acknowledgements}

This work was supported by the Ministry of Science and Technology under Grant No. MOST-103 -2112 -M-008-001MY2, MOST 104-2112-M-008 -013 -MY3, MOST 1052112-M-008 -003 -MY3, and MOST 1032112 M 008019 MY3.

\section{References}

1. C. D. Gregory, B. Loupias, J. Waugh, P. Barroso, S. Bouquet, E. Brambrink, S. Dono, E. Falize, J. Howe, Y. Kuramitsu, R. Kodama, M. Koenig, C. Michaut, S. Myers, W. Nazarov, M. M. Notley, A. Oya, S. Pikuz, M. Rabec le Gloahec, Y. Sakawa, C. Spindloe, M. Streeter, L. A. Wilson, and N. C. Woolsey, Plasma Phys. Control. Fusion 50, 124039 (2008).

2. Y. Kuramitsu, Y. Sakawa, J. N. Waugh, C. D. Gregory, T. Morita, S. Dono, H. Aoki, H. Tanji, B. Loupias, M. Koenig, N. Woolsey, and H. Takabe, Astrophys. J. Lett. 707, L137 (2009).

3. C. D. Gregory, B. Loupias, J. Waugh, S. Dono, S. Bouquet, E. Falize, Y. Kuramitsu, C. Michaut, W. Nazarov, S. A. Pikuz, Y. Sakawa, N. C. Woolsey, and M. Koenig, Phys. Plasmas 17, 052708 (2010).

4. A. Dizière, A. Pelka, A. Ravasio, B. Loupias, E. Falize, Y. Kuramitsu, Y. Sakawa, T. Morita, S. Pikuz, R. Yurchak, and M. Koenig, Phys. Plasmas 22, 012702 (2015).

5. T. Morita, Y. Sakawa, Y. Kuramitsu, S. Dono, H. Aoki, H. Tanji, T. N. Kato, Y. T. Li, Y. Zhang, X. Liu, J. Y. Zhong, H. Takabe, and J. Zhang, Phys. Plasmas 17, 122702 (2010).
6. Y. Kuramitsu, Y. Sakawa, T. Morita, J. N. Waugh, C. D. Gregory, S. Dono, H. Aoki, H. Tanji, M. Koenig, N. Woolsey, and H. Takabe, Phys. Rev. Lett. 106, 175002 (2011).

7. Y. Kuramitsu, Y. Sakawa, S. Dono, C. D. Gregory, S. A. Pikuz, B. Loupias, M. Koenig, J. N. Waugh, N. Woolsey, T. Morita, T. Moritaka, T. Sano, Y. Matsumoto, A. Mizuta, N. Ohnishi, and H. Takabe, Phys. Rev. Lett. 108, 195004 (2012).

8. Y. Kuramitsu, Y. Sakawa, T. Morita, T. Ide, K. Nishio, H. Tanji, H. Aoki, S. Dono, C. D. Gregory, J. N. Waugh, N. Woolsey, A. Diziere, A. Pelka, A. Ravasio, B. Loupias, M. Koenig, S. A. Pikuz, Y. T. Li, Y. Zhang, X. Liu, J. Y. Zhong, J. Zhang, G. Gregori, N. Nakanii, K. Kondo, Y. Mori, E. Miura, R. Kodama, Y. Kitagawa, K. Mima, K. A. Tanaka, H. Azechi, T. Moritaka, Y. Matsumoto, T. Sano, A. Mizuta, N. Ohnishi, M. Hoshino, and H. Takabe, Plasma Phys. Control. Fusion 54, 124049 (2012).

9. N. L. Kugland, D. D. Ryutov, P.-Y. Chang, R. P. Drake, G. Fiksel, D. H. Froula, S. H. Glenzer, G. Gregori, M. Grosskopf, M. Koenig, Y. Kuramitsu, C. Kuranz, M. C. Levy, E. Liang, J. Meinecke, F. Miniati, T. Morita, A. Pelka, C. Plechaty, R. Presura, A. Ravasio, B. A. Remington, B. Reville, J. S. Ross, Y. Sakawa, A. Spitkovsky, H. Takabe, and H.-S. Park, Nature Phys. 8, 809 (2012).

10. Y. Sakawa, T. Morita, Y. Kuramitsu, and H. Takabe, Adv. Phys. X 1, 425 (2016).

11. Y. Kuramitsu, N. Ohnishi, Y. Sakawa, T. Morita, H. Tanji, T. Ide, K. Nishio, C. D. Gregory, J. N. Waugh, N. Booth, R. Heathcote, C. Murphy, G. Gregori, J. Smallcombe, C. Barton, A. Dizière, M. Koenig, N. Woolsey, Y. Matsumoto, A Mizuta, T. Sugiyama, S. Matsukiyo, T. Moritaka, T. Sano, and H. Takabe, Phys. Plasmas 23, 032126 (2016).

12. Y. Kuramitsu, A. Mizuta, Y. Sakawa, H. Tanji, T. Ide, T. Sano, M. Koenig, A. Ravasio, A. Pelka, H. Takabe, C. D. Gregory, N. Woolsey, T. Moritaka, S. Matsukiyo, Y. Matsumoto, and N. Ohnishi, Astrophys. J. 828, 93 (2016).

13. Y. Kuramitsu, N. Nakanii, K. Kondo, Y. Sakawa, Y. Mori, E. Miura, K. Tsuji, K. Kimura, S. Fukumochi, M. Kashihara, T. Tanimoto, H. Nakamura, T. Ishikura, K. Takeda, M. Tampo, R. Kodama, Y. Kitagawa, K. Mima, K. A. Tanaka, M. Hoshino, and H. Takabe, Phys. Plasmas 18, 010701 (2011).

14. Y. Kuramitsu, N. Nakanii, K. Kondo, Y. Sakawa, Y. Mori, E. Miura, K. Tsuji, K. Kimura, S. Fukumochi, M. Kashihara, T. Tanimoto, H. Nakamura, T. Ishikura, K. Takeda, M. Tampo, R. Kodama, Y. Kitagawa, K. Mima, K. A. Tanaka, M. Hoshino, and H. Takabe, Phys. Rev. E 83, 026401 (2011).

15. Y. Kuramitsu, H.-H. Chu, L.-N. Hau, S.-H. Chen, Y.-L. Liu, C.-Y. Hsieh, Y. Sakawa, T. Hideaki, and J. Wang, High Energy Density Phys. 17, 198 (2015).

16. T.-S. Hung, C.-H. Yang, J. Wang, S.-Y. Chen, J.-Y. Lin, and H.-H. Chu, Appl. Phys. B, published online, 2014.

17. Y.-M. Chen, S.-M. He, C.-H. Huang, C.-C. Huang, W.-P. Shih, C.-L. Chu, J. Kong, J. Li, and C.-Y. Su, Nanoscale 8, 3555 (2016).

18. X. Li, W. Cai, J. An, S. Kim, J. Nah, D. Yang, R. Piner, A. Velamakanni, I. Jung, E. Tutuc, S. K. Banerjee, L. Colombo, and R. S. Ruoff, Science 324, 1312 (2009).

19. X. Li, C. W. Magnuson, A. Venugopal, J. An, J. W. Suk, B. Han, M. Borysiak, W. Cai, A. Velamakanni, Y. Zhu, L. Fu, E. M. Vogel, E. Voelkl, L. Colombo, and R. S. Ruoff, Nano Lett. 10, 4328 (2010).

20. J. M. Wofford, S. Nie, K. F. McCarty, N. C. Bartelt, and O. D. Dubon, Nano Lett. 10, 4890 (2010).

21. T. Esirkepov, M. Borghesi, S. V. Bulanov, G. Mourou, and T. Tajima, Phys. Rev. Lett. 92, 175003 (2004). 
22. A. Macchi, F. Cattani, T. V. Liseykina, and F. Cornolti, Phys. Rev. Lett. 94, 165003 (2005).

23. A. Henig, S. Steinke, M. Schnürer, T. Sokollik, R. Hörlein, D. Kiefer, D. Jung, J. Schreiber, B. M. Hegelich, X. Q. Yan, J. Meyer-Ter-Vehn, T. Tajima, P. V. Nickles, W. Sandner, and D. Habs, Phys. Rev. Lett. 103, 245003 (2009).

24. S. Kar, K. F. Kakolee, B. Qiao, A. Macchi, M. Cerchez, D. Doria, M. Geissler, P. McKenna, D. Neely, J. Osterholz, R. Prasad, K. Quinn, B. Ramakrishna, G. Sarri, O. Willi, X. Y. Yuan, M. Zepf, and M. Borghesi, Phys. Rev. Lett. 109, 185006 (2012).

25. T.-C. Liu, X. Shao, C.-S. Liu, B. Eliasson, J. Wang, and S.-H. Chen, Phys. Plasmas 20, 103112 (2013).
26. G. M. Petrov, C. McGuffey, A. G. R. Thomas, K. Krushelnick, and F. N. Beg., J. Appl. Phys. 119, 053302 (2016).

27. I. Jong Kim, K. H. Pae, I. W. Choi, C.-L. Lee, H. T. Kim, H. Singhal, J. H. Sung, S. K. Lee, H. W. Lee, P. V. Nickles, T. M. Jeong, C. M. Kim, and C. H. Nam, Phys. Plasmas 23, 070701 (2016).

28. M.-C. Chuang and W.-Y. Woon, Carbon 103, 384 (2016).

29. Y. Wang, Y. Zheng, X. Xu, E. Dubuisson, Q. Bao, J. Lu, and K. P. Loh, ACS Nano 5, 9927 (2011).

30. L. Gao, W. Ren, H. Xu, L. Jin, Z. Wang, T. Ma, L.-P. Ma, Z. Zhang, Q. Fu, L.-M. Peng, X. Bao, and H.-M. Cheng, Nat. Commun. 3, 699 (2012). 